\title{
Small Cavity of Left Ventricle Does Not Affect Short-term Outcome in Patients with Rheumatic Mitral Valve Stenosis Undergoing Mitral Valve Replacement
}

\author{
Junnan Zheng, MD*, Tingting Tao, MD*, Yiming Ni, MD, Liang Ma, MD, Haige Zhao, MD \\ Department of Cardiovascular Surgery, the First Affiliated Hospital, College of Medicine, Zhejiang University, Hangzhou, China
}

\section{ABSTRACT}

Background: Small cavity left ventricle (SCLV) may affect the clinical outcomes of patients undergoing mitral valve replacement (MVR). This study aims to investigate the incidence of SCLV in patients with rheumatic mitral valve stenosis undergoing MVR and analyze its effect on short-term patient outcomes.

Methods: We retrospectively examined all consecutive patients with isolated or concomitant MVR for rheumatic mitral valve stenosis in our center from 2013 to 2018. SCLV was defined as end-diastolic volume index $\leq 50 \mathrm{ml} / \mathrm{m} 2$. After inclusion and exclusion, a total of 1,437 patients were analyzed. The baseline information was collected and compared between SCLV and non-SCLV patients. Multivariate logistic regression analysis was conducted to determine the effect of SCLV on early mortality.

Results: A total of 1,437 patients were included in the study. SCLV was detected in $13.57 \%$ of the patients. Compared with the non-SCLV group, patients with SCLV were smaller-sized and primarily female. There were no significant differences between SCLV and non-SCLV patients regarding major postoperative complications, nor were there incidence of prosthesis-patient mismatch. Logistic regression analysis showed that SCLV was not a risk factor for short-term mortality $(P=0.998)$.

Conclusions: Our results demonstrated that SCLV was not associated with poorer early outcomes after MVR surgery in patients with rheumatic mitral valve stenosis.

\section{INTRODUCTION}

Rheumatic heart diseases are known as the dominating causes of mitral valve diseases in developing countries, such as China [Chandrashekhar 2009]. Untreated severe rheumatic mitral stenosis can lead to a very poor prognosis, including death; thus, interventional treatment is strongly recommended [Horstkotte 1991].

Received September 17, 2020; accepted November 1, 2020

* Junnan Zheng and Tingting Tao contributed equally to this study.

Correspondence: Haige Zhao, Department of Cardiovascular Surgery, First Affiliated Hospital, College of Medicine, Zhejiang University, 79\# Qingchun Road, Hangzhou, China, 310000, Telephone +860571 87236841 (e-mail: baigezhao@zju.edu.cn)
Currently, the mainstream treatment of rheumatic mitral valve stenosis includes percutaneous balloon valvuloplasty and mitral valve replacement (MVR). Thanks to its better long-term result and well-controlled morbidity/mortality, MVR has become the treatment of choice in severe rheumatic mitral valve stenosis or regurgitation, particularly in elderly patients [Harb 2017].

It has been reported that a substantial proportion of patients with rheumatic mitral valve stenosis were characterized with a small cavity left ventricle (SCLV) by echocardiography [Jiang 2009]. While it has been well clarified that an enlarged left ventricle is associated with adverse outcome in patients undergoing MVR [Wenbin 2002; Zile 1993], the effect of SCLV on patient outcomes after MVR has only been partially explored.

However, several reports did propose that SCLV may indeed lead to adverse postoperative outcomes. Small left ventricle size was reported to be a significant risk factor for left ventricle rupture after MVR [Hosono 2008]. Moreover, one report suggested that SCLV could be a risk factor for left ventricular outflow tract obstruction after MVR [Jett 1986]. A further study showed that patients with SCLV were at higher risk for surgery with and increasing risk of prosthesispatient mismatch (PPM) [Popov 2013]. However, our previous research demonstrated that, although mitral PPM was associated with small left ventricle, it was not associated with poorer early- and mid-term outcomes after MVR in patients with rheumatic mitral valve stenosis [Akuffu 2018].

Therefore, we aim to investigate the incidence of SCLV in patients with rheumatic mitral valve stenosis in our center and analyze whether SCLV was associated with adverse outcomes after MVR in this population.

\section{METHODS}

Patient population and data collection: We retrospectively analyzed data of all consecutive patients who underwent isolated or concomitant MVR at our center from January 2013 to December 2018. All patients received MVR at our center and were not amenable to balloon valvuloplasty, assessed by cardiologists. After obtaining written informed consent waived by the Hospital Review Board, we reviewed data of patients aged 18 years or older who were undergoing isolated MVR or MVR concomitant with other open-heart procedures. Patients who received MVR due to non-rheumatic causes, pure mitral valve regurgitation, failed mitral 


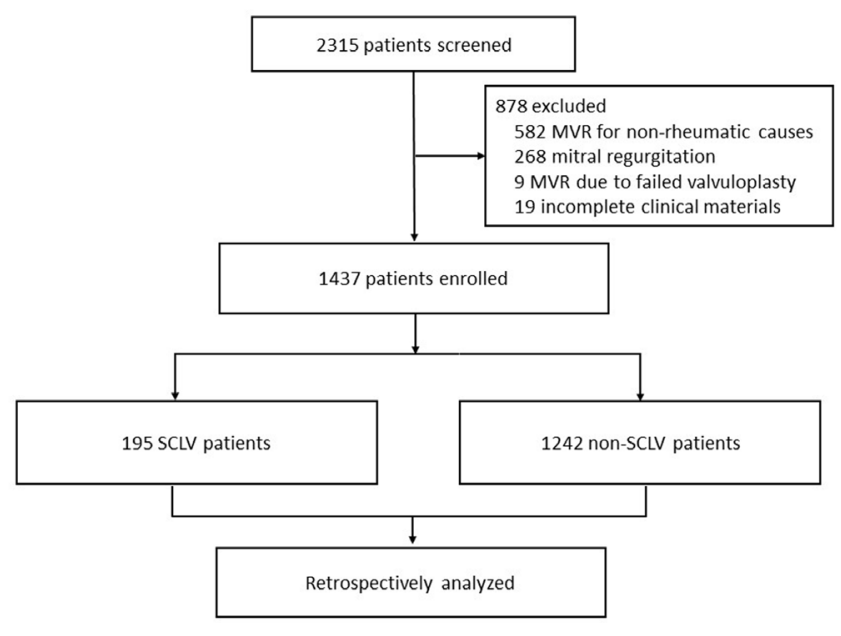

Figure 1. Consort flow diagram of patient enrollment

valvuloplasty, and patients with incomplete clinical data were excluded. (Figure 1) Altogether, 1,437 patients were included in this study. Baseline, intraoperative, and outcome data were prospectively collected and validated, which had been queried retrospectively.

SCLV, PPM, and definitions: According to previous references, SCLV was defined as end-diastolic volume index (EDVi) $\leq 50 \mathrm{ml} / \mathrm{m}^{2}$ during the hospitalization period [Abbo 1994; Gomez-Duran 1984]; thus, patients with EDVi > $50 \mathrm{ml} /$ $\mathrm{m} 2$ were listed as the non-SCLV group. EDVi was obtained with end-diastolic volume (EDV) divided by the body surface area (BSA) of the patient. EDVs were preoperatively derived by non-contrast transthoracic echocardiogram by using Simpson's disk method in the apical 4- and 2-chamber views. BSA was calculated using the Dubois formula. PPM was defined according to the value of the effective orifice area index (EOAi), which was obtained with effective orifice area
(EOA) divided by the BSA of the patient. Mitral PPM was defined as EOAi $\leq 1.2 \mathrm{~cm}^{2} / \mathrm{m}^{2}$. EOAi $\leq 0.9 \mathrm{~cm}^{2} / \mathrm{m}^{2}$ was considered severe mitral PPM. BSA was calculated using the Dubois formula and EOA of the mitral valve prosthesis was derived from in vitro measurements provided by the manufacturers and from scientific publications, as outlined in Table 1. (Table 1)

Surgical technique: A total of 1,242 mechanical prostheses and 195 bioprostheses were implanted. Prostheses used were: mechanical prosthesis (CarboMedics Orbis Universal, CarboMedics, Inc., Austin, TX, USA) $(N=741)$, St. Jude Master (St. Jude Medical, Inc., St. Paul, MN, USA) $(N=363)$, ATS open pivot (ATS Medical, Inc., Minneapolis, MN, USA) $(N=138)$, bioprosthesis (Hancock II Porcine Bioprosthesis, Medtronic, Inc., Minneapolis, MN, USA) $(N=83)$, Mosaic Porcine Bioprosthetic Valves (Medtronic, Inc., Minneapolis, MN, USA) $(N=10)$, Bicor Stented Bioprosthesis (St. Jude Medical, Inc., St. Paul, MN, USA) $(N=41)$, CarpentierEdwards Perimount (Baxter Healthcare Corp., Edwards Division, Santa Ana, CA, USA) $(N=61)$.

Concomitant procedures included aortic valve replacement, tricuspid annuloplasty, coronary artery bypass grafting, atrial septum defect repair, atrial fibrillation radiofrequency ablation, and/or other open-heart procedures. Standard anesthesia and cardiopulmonary bypass strategies were implemented. The patients were approached either through a full median sternotomy (1,356 patients, $94.4 \%)$ or through a right thoracotomy (81 patients, $5.6 \%$ ) followed by an antegrade $4: 1$ cold blood cardioplegia for myocardial protection. Intermittent antegrade plus continuous retrograde cardioplegia was applied for patients with coronary stenosis. Antegrade cold blood cardioplegia perfusion was maintained at a frequency of once every 20 minutes.

The patients were preoperatively consulted regarding the choice of prostheses. However, the final decision of the type and the size of prosthesis was made by the surgeons during operation, taking into consideration the preoperative

Table 1. In vivo EOA value $\left(\mathrm{cm}^{2}\right)$ of each valve prothesis

\begin{tabular}{|c|c|c|c|c|c|c|c|c|}
\hline \multicolumn{9}{|l|}{ Mechanical } \\
\hline St. Jude Master & & 1.0 & 1.5 & 1.8 & 1.8 & 2.0 & 2.0 & $\begin{array}{l}\text { Bitar 1995; } \\
\text { Magne } 2007\end{array}$ \\
\hline Medtronic Hancock II & & - & 1.5 & 1.8 & 1.9 & 2.6 & 2.6 & Lam 2007 \\
\hline Medtronic Mosaic & & - & 1.5 & 1.7 & 1.9 & 1.9 & - & Magne 2007 \\
\hline St. Jude Bicor Stented & & - & 1.4 & 1.5 & 2.3 & 2.2 & 2.3 & $\begin{array}{c}\text { Borracci } \\
2016\end{array}$ \\
\hline
\end{tabular}


information and intraoperative findings. When performing MVR, native posterior leaflet and sub-valvular structures were preserved as much as possible.

Statistical analysis: The Kolmogorov-Smirnov test was used to verify the distribution of all the quantitative variables. Variables that fit the Gaussian distribution were presented as mean \pm standard deviation (SD), while non-Gaussian distributed variables were presented as medians (interquartile range). Categorical variables were expressed as absolute number (percentage). Pearson's $\chi 2$ test was used for descriptive, binary multivariate statistics, such as the comparison of portions, while the Student's unpaired t-test was used for normally distributed data comparisons. The Mann-Whitney U test was otherwise used for comparison of non-Gaussian distributed variables. Two-tailed $P$-values were derived from the calculated test statistics, and $P \leq$ 0.05 was considered statistically significant. Binary multivariate logistic regression analysis was performed to study the factors affecting postoperative mortality. SPSS Statistics 23.0 software (IBM, Armonk, NY, USA) was used to analyze the data.

\section{RESULTS}

Table 2. Preoperative patient baseline information
SCLV and preoperative patient baseline information: After inclusion and exclusion (Figure 1), a total of 1,437 patients were included in this study. SCLV was detected in $13.572 \%(195 / 1,437)$ of the patients. Preoperative patient baseline information was listed in Table 2. (Table 2) Compared with the non-SCLV group, patients with SCLV were smaller-sized and primarily female. They had a lower prevalence smoking history. The baseline characteristics of the two groups were comparable.

Operative data: As demonstrated in Table 3, there were no significant differences between SCLV and non-SCLV patients regarding cardiac surgery history, cardiopulmonary bypass (CPB) time, and combined procedures. (Table 3) However, the cross-clamp time of SCLV patients was shorter. Size distribution in both mechanical prosthesis and bioprosthesis was analyzed (Table 4). (Table 4) Small-sized prostheses (25\# or smaller) were more used in SCLV patients, while large-sized prostheses (27\# or larger) were more used in nonSCLV patients.

Postoperative outcomes and factors affecting postoperative mortality: There were no obvious differences between SCLV and non-SCLV patients regarding early postoperative complications, including blood transfusion,

\begin{tabular}{|c|c|c|c|c|}
\hline Age, yr. & $54(47-61)$ & $53(46-60)$ & $54(48-61)$ & 0.092 \\
\hline $\mathrm{BMI}, \mathrm{kg} / \mathrm{m}^{2}$ & $22.56 \pm 3.343$ & $22.47 \pm 2.95$ & $22.58 \pm 3.50$ & 0.652 \\
\hline BSA, $\mathrm{m}^{2}$ & $1.56 \pm 0.16$ & $1.54 \pm 0.15$ & $1.57 \pm 0.16$ & 0.017 \\
\hline Diabetes & $156(11.8 \%)$ & $21(10.8 \%)$ & $135(10.9 \%)$ & 0.967 \\
\hline Hypertension & $215(15.0 \%)$ & $22(11.3 \%)$ & $193(15.5 \%)$ & 0.121 \\
\hline Cerebrovascular accident & $91(6.3 \%)$ & $15(7.7 \%)$ & $76(6.1 \%)$ & 0.402 \\
\hline Coronary heart disease & $41(2.9 \%)$ & $3(1.5 \%)$ & $38(3.0 \%)$ & 0.236 \\
\hline NYHA functional class ( $\geq \mathrm{III})$ & $453(31.5 \%)$ & $66(33.8 \%)$ & $387(31.2 \%)$ & 0.453 \\
\hline LVDD, mm & $49.17 \pm 7.94$ & $38.29 \pm 4.13$ & $50.88 \pm 6.99$ & $<0.001$ \\
\hline $\mathrm{EDV}, \mathrm{ml}$ & $133.70 \pm 73.34$ & $60.26 \pm 15.76$ & $145.24 \pm 72.14$ & $<0.001$ \\
\hline$E D V i, m l / m^{2}$ & $85.18 \pm 44.68$ & $39.17 \pm 9.52$ & $92.41 \pm 43.71$ & $<0.001$ \\
\hline LAD, mm & $52.19 \pm 11.49$ & $49.12 \pm 10.25$ & $52.68 \pm 11.60$ & $<0.001$ \\
\hline Emergency surgery & $4(0.3 \%)$ & $1(0.5 \%)$ & $3(0.2 \%)$ & 0.504 \\
\hline Aspirin within 5 days & $4(0.3 \%)$ & $2(1.0 \%)$ & $3(0.2 \%)$ & 0.084 \\
\hline Clopidogrel within 5 days & $1(0.1 \%)$ & $0(0.0 \%)$ & $1(0.1 \%)$ & 0.692 \\
\hline
\end{tabular}

SCLV, small cavity left ventricle; BMI, body mass index; BSA, body surface area; NYHA, New York Heart Association; MI, myocardial infarction; LVEF, left ventricular ejection fraction; LVDD, left ventricular diastolic diameter; LAD, left atrial diameter; EDV, end-diastolic volume 
Table 3. Intraoperative data

\begin{tabular}{|c|c|c|c|c|}
\hline CPB time, $\min$ & $85(71-93)$ & $81(70-90)$ & $86(71-93)$ & 0.083 \\
\hline Mechanical prosthesis & $1242(86.4 \%)$ & $174(89.2 \%)$ & $1068(85.5 \%)$ & 0.219 \\
\hline Bioprosthesis & $195(13.6 \%)$ & $21(10.8 \%)$ & $174(14.0 \%)$ & 0.219 \\
\hline AVR & $687(47.8 \%)$ & $81(41.5 \%)$ & $606(48.8 \%)$ & 0.059 \\
\hline TVP & $594(41.3 \%)$ & $79(40.5 \%)$ & $515(41.5 \%)$ & 0.802 \\
\hline CABG & $41(2.9 \%)$ & $3(1.5 \%)$ & $38(3.0 \%)$ & 0.230 \\
\hline AFRA or MAZE & $130(9.0 \%)$ & $20(10.2 \%)$ & $110(8.8 \%)$ & 0.526 \\
\hline Others & $164(11.4 \%)$ & $21(10.8 \%)$ & $143(11.5 \%)$ & 0.761 \\
\hline
\end{tabular}

SCLV, small cavity left ventricle; CPB, cardiopulmonary bypass; CABG, coronary artery bypass grafting surgery; AVR, aortic valve replacement; TVP, tricuspid valve plasty; AFRA, atrial fibrillation radio frequency surgery; MAZE, cox-maze or mini maze surgery

Table 4. Distribution of size and type of prostheses

\begin{tabular}{lccc}
\hline Size and prosthesis type & SCLV group $(N=195)$ & non-SCLV group $(N=1242)$ & $P$-value \\
\hline Mechanical prosthesis $(N=1242)$ & $174(89.2 \%)$ & $1068(86.0 \%)$ & 0.219 \\
$23 \mathrm{~mm}$ & $2(1.1 \%)$ & $3(0.3 \%)$ & 0.093 \\
$25 \mathrm{~mm}$ & $77(44.2 \%)$ & $292(27.3 \%)$ & $<0.001$ \\
$27 \mathrm{~mm}$ & $84(48.3 \%)$ & $684(64.0 \%)$ & -0.001 \\
$29 \mathrm{~mm}$ & $11(6.3 \%)$ & $85(8.0 \%)$ & 0.453 \\
$31 \mathrm{~mm}$ & - & $4(0.4 \%)$ & - \\
Bioprosthesis $(N=195)$ & $21(10.8 \%)$ & $174(14.0 \%)$ & 0.219 \\
$25 \mathrm{~mm}$ & $12(57.1 \%)$ & $44(25.3 \%)$ & 0.002 \\
$27 \mathrm{~mm}$ & $9(42.9 \%)$ & $122(70.1 \%)$ & - \\
$29 \mathrm{~mm}$ & - & $7(4.0 \%)$ & - \\
$31 \mathrm{~mm}$ & - & $1(0.6 \%)$ & - \\
\hline
\end{tabular}

SCLV, small cavity left ventricle

ventilation time, reintubation, intensive care unit (ICU) time, postop stroke, postop atrial fibrillation, PPM, and short-term mortality. (Table 5) Although the overall occurrence of PPM did not differ significantly between SCLV and non-SCLV patients, the rate of severe PPM was higher in the SCLV group (3.6\% vs. $1.3 \%, P=0.017)$. Also, there was a small increase in hospitalization expense for the non-SCLV patients. What's more, 22 patients underwent reoperation, but none of these reoperations were due to SCLV related left ventricular outflow tract obstruction or rupture of the left ventricle.

Altogether, eight $(0.6 \%)$ patients died within 30 days after surgery. Among them, four patients died due to malignant arrhythmia or cardiac arrest, two patients died of severe systemic infection, one patient died of uncontrollable bleeding, and one patient died of stroke. Among these deaths, three patients had SCLV.

Logistic regression analysis showed that low preoperative LVEF and previous cardiac surgery were independent factors predicting postoperative short-term all-cause mortality. However, SCLV, along with overall PPM, was not a risk factor for short-term mortality. (Table 6)

\section{DISCUSSION}

SCLV occurrence and patient characteristics: The incidence of SCLV in our single-centered cohort was $13.57 \%$. A 
Table 5. Postoperative outcomes

\begin{tabular}{|c|c|c|c|c|}
\hline Perioperative transfusion & $482(33.5 \%)$ & $59(30.2 \%)$ & $423(34.0 \%)$ & 0.296 \\
\hline Reintubation & $5(0.3 \%)$ & $0(0.0 \%)$ & $5(0.4 \%)$ & 0.375 \\
\hline Duration of first time ICU, hr. & $72(72-96)$ & $72(72-96)$ & $72(72-96)$ & 0.768 \\
\hline Chest tube output, $\mathrm{ml}$ & $508.8 \pm 280.8$ & $504.3 \pm 306.3$ & $509.5 \pm 276.7$ & 0.824 \\
\hline Reoperation for bleeding & $22(1.5 \%)$ & $5(2.6 \%)$ & $17(1.4 \%)$ & 0.206 \\
\hline Surgical wound infection & $3(0.2 \%)$ & $0(0.0 \%)$ & $3(0.2 \%)$ & 0.492 \\
\hline Stroke & $9(0.6 \%)$ & $3(1.5 \%)$ & $6(0.5 \%)$ & 0.082 \\
\hline Newly onset AF & $1(0.1 \%)$ & $0(0.0 \%)$ & $1(0.1 \%)$ & 0.692 \\
\hline Hospitalization expense, USD & $13275(11460-15448)$ & $12818(10901-14957)$ & 13334(11526-15506) & 0.020 \\
\hline Length of stay, $d$ & $14(12-17)$ & $14(12-17)$ & $14(12-17)$ & 0.411 \\
\hline
\end{tabular}

SCLV, small cavity of left ventricle; ICU, intensive care unit; AF, atrial fibrillation; PPM prosthesis-patient mismatch

Table 6. Logistic regression analysis for post-operative short-term mortality

\begin{tabular}{|c|c|c|c|c|}
\hline Preoperative LVEF & 62.31 & 0.787 & $0.637-0.972$ & 0.026 \\
\hline SCLV & $13.6 \%$ & - & - & 0.998 \\
\hline PPM & $34.0 \%$ & - & - & 0.996 \\
\hline
\end{tabular}

$\mathrm{Cl}$, confidence interval; LVEF, left ventricular ejection fraction; SCLV, small cavity of left ventricle; PPM, prosthesis-patient mismatch

previous study from Popov et al., 2013, using EDV $\leq 75 \mathrm{ml}$ as a cut-off value for SCLV, reported incidences of SCLV to be about $7.0 \%$ in patients undergoing MVR in a Ukrainian population [Popov 2013]. In the Asian population, especially the Eastern Chinese population, mitral stenosis was considered to occur more frequently than in western populations, due to rheumatic causes. While mitral stenosis has been proven to be associated with compromised left ventricle compliance and reduced ventricular chamber size, due to cardiac remodeling (smallness) and mechanical constraint [Liu 1992], it is not hard to conclude that the SCLV incidence in this study population is sure to be higher.

It was also found that there were more female patients in the SCLV group (79\% female), consistent with previous studies [Kou 2014; Popov 2013]. This also helped explain the smaller body size of SCLV patients and the lower smoking history of SCLV patients since smokers in China were primarily male.

Moreover, it was noticed that the average LVEF in SCLV patients was slightly higher than in non-SCLV patients although not significantly $(63.23 \pm 7.86$ vs. $62.17 \pm 8.42, P=$ $0.100)$. It has long been demonstrated that mitral stenosis, along with its restricted left ventricular compliance, does not affect the systolic function of the left ventricle [Gash 1983]. However, the increased left ventricular preload, indicated by the larger end-diastolic volume of the non-SCLV patients, would to some extent cause the reduced ejection performance [Mohan 1990].

Intraoperative data showed that the cross-clamp time was shorter in the SCLV group. This might be explained because less time was spent suturing the mitral prosthesis due to the smaller mitral annulus diameter of the SCLV patients.

SCLV and patient outcome: Our results showed that SCLV did not affect major early postoperative complications 
or short-term mortality. Although $8(0.6 \%)$ patients died during the short-term postoperative period, left ventricle rupture and left ventricular outflow tract obstruction related to SCLV was not detected. Logistic regression analysis showed several independent factors predicting postoperative shortterm all-cause mortality, whereas SCLV was not one of them, and PPM as well did not affect short-term mortality. In western populations, where degenerative mitral regurgitation is the major etiology, the mitral annulus is often large. In these patients, when SCLV occurs, the left ventricle compliance will further be compromised, adding to an increasing risk of left ventricle rupture that most often leads to postoperative death. However, in patients with rheumatic mitral stenosis, SCLV often is accompanied with a small-sized mitral annulus and the left ventricle compliance remains. Thus, the risk of left ventricle rupture is limited.

Clinical implication for East Asian population: In East Asian populations, the dominating causes for mitral valve diseases were rheumatic causes. For this group of patients, mitral stenosis was more common and SCLV remained at a high incidence. However, the body surface area of these patients was also smaller, therefore, the incidence of PPM might not be significantly elevated. In this study, results showed that the overall occurrence of PPM did not differ significantly between SCLV and non-SCLV patients, but the rate of severe PPM was higher in the SCLV group. However, our previous research demonstrated that although mitral PPM was associated with small left ventricle, it was not associated with poorer early- and mid-term outcomes after MVR in patients with rheumatic mitral valve disease [Akuffu 2018]. As results of this study also showed that SCLV did not increase adverse outcomes following MVR, we did not suggest an aggressive implantation of larger-sized mitral prostheses in these patients to avoid PPM. Aggressive implantation of a largesized prosthesis by excessive excision of chordae and subvalvular apparatus might compromise the effective cardiac muscular contraction of the left ventricle, leading to left ventricular systolic dysfunction or, even worse, add to the risk of left ventricular rupture.

Limitations of the study: There are several limitations of the study that must be declared. First, this is a retrospective analysis. As an inherent disadvantage, the recorded differences in patient outcomes could have originated from smaller recorded or unrecorded differences between SCLV and nonSCLV patients. Second, EOA was predicted by reference tables, which may not reflect the actual in vivo values of the EOAi in defining PPM. Moreover, this is a single-centered short-term study, and the sample size and follow-up time were limited. Therefore, a randomized prospective multicentered clinical trial is needed for further evaluation of the effects of SCLV.

\section{CONCLUSION}

Our results demonstrated that SCLV was not associated with poorer early outcomes after MVR surgery in patients with rheumatic mitral valve diseases. An aggressive implantation of a large prosthesis should carefully be weighed before implementation.

\section{ACKNOWLEDGEMENTS}

This study is funded by 1) Postdoctoral Research Foundation of China (Project No.: 2018M640566); 2) Medicine and Health Science and Technology Plan Projects of Zhejiang Province for young scholars (Project No.: 2019RC157); and 3) Natural Science Foundation of Zhejiang Province (Project No: LQ20H020004).

\section{REFERENCES}

Abbo KM, Carroll JD. 1994. Hemodynamics of mitral stenosis: A review. Cathet Cardiovasc Diagn Suppl. 2:16-25.

Akuffu AM, Zhao H, Zheng J, Ni Y. 2018. Prosthesis-patient mismatch after mitral valve replacement: A single-centered retrospective analysis in east china. J Cardiothorac Surg. 13:100.

Bitar JN, Lechin ME, Salazar G, Zoghbi WA. 1995. Doppler echocardiographic assessment with the continuity equation of St. Jude medical mechanical prostheses in the mitral valve position. Am J Cardiol. 76:287-93.

Borracci RA, Rubio M, Sestito ML, Ingino CA, Barrero C, Rapallo CA. 2016. Incidence of prosthesis-patient mismatch in patients receiving mitral biocor(r) porcine prosthetic valves. Cardiol J. 23:178-83.

Chandrashekhar Y, Westaby S, Narula J. 2009. Mitral stenosis. Lancet 374:1271-83.

Cohn LH. 2012. Cardiac surgery in the adult. 4th ed. New York: McGraw-Hill Medical.

Gash AK, Carabello BA, Cepin D, Spann JF. 1983. Left ventricular ejection performance and systolic muscle function in patients with mitral stenosis. Circulation. 67:148-54.

Gomez-Duran C. 1984. Recent progress in mitral valve disease. London: Butterworths.

Harb SC, Griffin BP. 2017. Mitral valve disease: A comprehensive review. Curr Cardiol Rep. 19:73.

Horstkotte D, Niehues R, Strauer BE. 1991. Pathomorphological aspects, aetiology and natural history of acquired mitral valve stenosis. Eur Heart J. 12 Suppl B:55-60.

Hosono M, Shibata T, Sasaki Y, et al. 2008. Left ventricular rupture after mitral valve replacement: Risk factor analysis and outcome of resuscitation. J Heart Valve Dis. 17:42-7.

Jett GK, Jett MD, Bosco P, van Rijk-Swikker GL, Clark RE. 1986. Left ventricular outflow tract obstruction following mitral valve replacement: Effect of strut height and orientation. Ann Thorac Surg. 42:299-303.

Jiang SL, Gao CQ, Li BJ, et al. 2009. [structural and functional changes of postoperative small left ventricle patients]. Zhonghua Wai Ke Za Zhi. 47:924-6.

Kou S, Caballero L, Dulgheru R, et al. 2014. Echocardiographic reference ranges for normal cardiac chamber size: Results from the norre study. Eur Heart J Cardiovasc Imaging. 15:680-90.

Lam BK, Chan V, Hendry P, et al. 2007. The impact of patient-prosthesis 
mismatch on late outcomes after mitral valve replacement. J Thorac Cardiovasc Surg. 133:1464-73.

Liu CP, Ting CT, Yang TM, et al. 1992. Reduced left ventricular compliance in human mitral stenosis. Role of reversible internal constraint. Circulation. 85:1447-56.

Magne J, Mathieu P, Dumesnil JG, et al. 2007. Impact of prosthesispatient mismatch on survival after mitral valve replacement. Circulation. 115:1417-25.

Mohan JC, Chutani SK, Sethi KK, Arora R, Khalilullah M. 1990.
Determinants of left ventricular function in isolated rheumatic mitral stenosis. Indian Heart J. 42:175-9.

Popov V, Shimon V, Yu L. 2013. Mitral valve replacement with small cavity of left ventricle. Journal of Cardiothoracic Surgery. 8:1-.

Wenbin L, Jianqun Z, Qiwen Z, et al. 2002. Surgical treatment of mitral regurgitation associated with enlarged left ventricle. J Heart Valve Dis. 11:181-4.

Zile MR, Tomita M, Ishihara K, et al. 1993. Changes in diastolic function during development and correction of chronic lv volume overload produced by mitral regurgitation. Circulation. 87:1378-88. 